\title{
Netrin1/DCC signaling promotes neuronal migration in the dorsal spinal cord
}

\author{
Harald J. Junge ${ }^{1^{*}}$, Andrea R. Yung ${ }^{2}$, Lisa V. Goodrich ${ }^{2}$ and Zhe Chen ${ }^{1^{*}}$ (D)
}

\begin{abstract}
Background: Newborn neurons often migrate before undergoing final differentiation, extending neurites, and forming synaptic connections. Therefore, neuronal migration is crucial for establishing neural circuitry during development. In the developing spinal cord, neuroprogenitors first undergo radial migration within the ventricular zone. Differentiated neurons continue to migrate tangentially before reaching the final positions. The molecular pathways that regulate these migration processes remain largely unknown. Our previous study suggests that the DCC receptor is important for the migration of the dorsal spinal cord progenitors and interneurons. In this study, we determined the involvement of the Netrin1 ligand and the $\mathrm{ROBO} 3$ coreceptor in the migration.

Results: By pulse labeling neuroprogenitors with electroporation, we examined their radial migration in Netrin 1 (Ntn1), Dcc, and Robo3 knockout mice. We found that all three mutants exhibit delayed migration. Furthermore, using immunohistochemistry of the BARHL2 interneuron marker, we found that the mediolateral and dorsoventral migration of differentiated dorsal interneurons is also delayed. Together, our results suggest that Netrin1/DCC signaling induce neuronal migration in the dorsal spinal cord.

Conclusions: Netrin1, DCC, and ROBO3 have been extensively studied for their functions in regulating axon guidance in the spinal commissural interneurons. We reveal that during earlier development of dorsal interneurons including commissural neurons, these molecules play an important role in promoting cell migration.
\end{abstract}

Keywords: Neuronal migration, Netrin1, DCC, ROBO3, Spinal cord neurons

\section{Background}

During spinal cord development, neuroprogenitors are born in the superficial layer of the ventricular zone (VZ) and undergo radial migration toward the lateral spinal cord [1-3]. Upon neurogenesis, neuroprogenitors exit the cell cycle and migrate out of the VZ. The postmitotic neurons continue to migrate along both mediolateral and dorsoventral axes as they differentiate into mature neurons. Different classes of interneurons, including contralateral- and ipsilateral-projecting neurons, are generated from progenitors in the dorsal spinal cord. Additional populations of interneurons as well as motor neurons arise from neuroprogenitors in the ventral half. Discrete neuronal populations that are located at different dorsoventral and mediolateral positions have stereotypical axonal projections and specific synaptic partners later during development [1-3]. Given the importance

\footnotetext{
* Correspondence: harald.junge@colorado.edu; zhe.chen@colorado.edu ${ }^{1}$ Department of MCDB, University of Colorado, Boulder, CO 80309, USA Full list of author information is available at the end of the article
}

of the migration in building the spinal cord circuitry, it is critical to identify the molecular mechanisms that regulate the radial and tangential migration of spinal cord neurons.

Recently, we found that in the knockout (KO) mice of the $D c c$ (deleted in colorectal carcinoma) receptor, the radial migration of dorsal progenitors and the tangential migration of differentiated dorsal interneurons are both delayed [4]. These migration defects may contribute to the axonal growth and guidance defects in the mutant during later stages of development [4]. In addition, Dcc KO has been previously shown to reduce the ventral migration of several classes of dorsal spinal cord interneurons [5]. These findings together prompted us to identify the molecular pathway that DCC acts upon during the migration.

The secreted Netrin proteins are conserved ligands for DCC from C. elegans to mammals [6]. Netrin/DCC are important for various neurodevelopmental processes, including axon guidance, neuronal migration, and synapse 
formation [7-9]. Within the contralateral-projecting commissural axons, Netrin1/DCC signaling induces axonal outgrowth and promotes axonal attraction [10-12]. By immunohistochemistry using specific antibodies, Netrin1 protein has been shown to be enriched at the ventral midline and at the lateral margin of the spinal cord in both chickens and mice $[13,14]$. The enrichment of Netrin1 at the ventral spinal cord is consistent with its role in attracting DCC-expressing commissural axons to the midline $[13,14]$. The presence of Netrin 1 at the dorsal lateral margin confines central axons within the CNS [15], and also inhibits abnormal entry by periphery sensory axons [16]. Whether Netrin1 can also attract the lateral and ventral migration of spinal cord neurons remains unknown.

ROBO3 is a member of the ROBO (roundabout) family of receptors for the SLIT proteins. While mammalian $\mathrm{ROBO} 1$ and $\mathrm{ROBO} 2$ mediate repulsion in commissural axons, $\mathrm{ROBO} 3$ inhibits $\mathrm{ROBO} 1 / 2$ and thus represses repulsion as commissural axons approach the midline [17]. In addition, $\mathrm{ROBO} 3$ has been shown to interact with DCC and function as a Netrin1 coreceptor to potentiate commissural axonal outgrowth and attraction

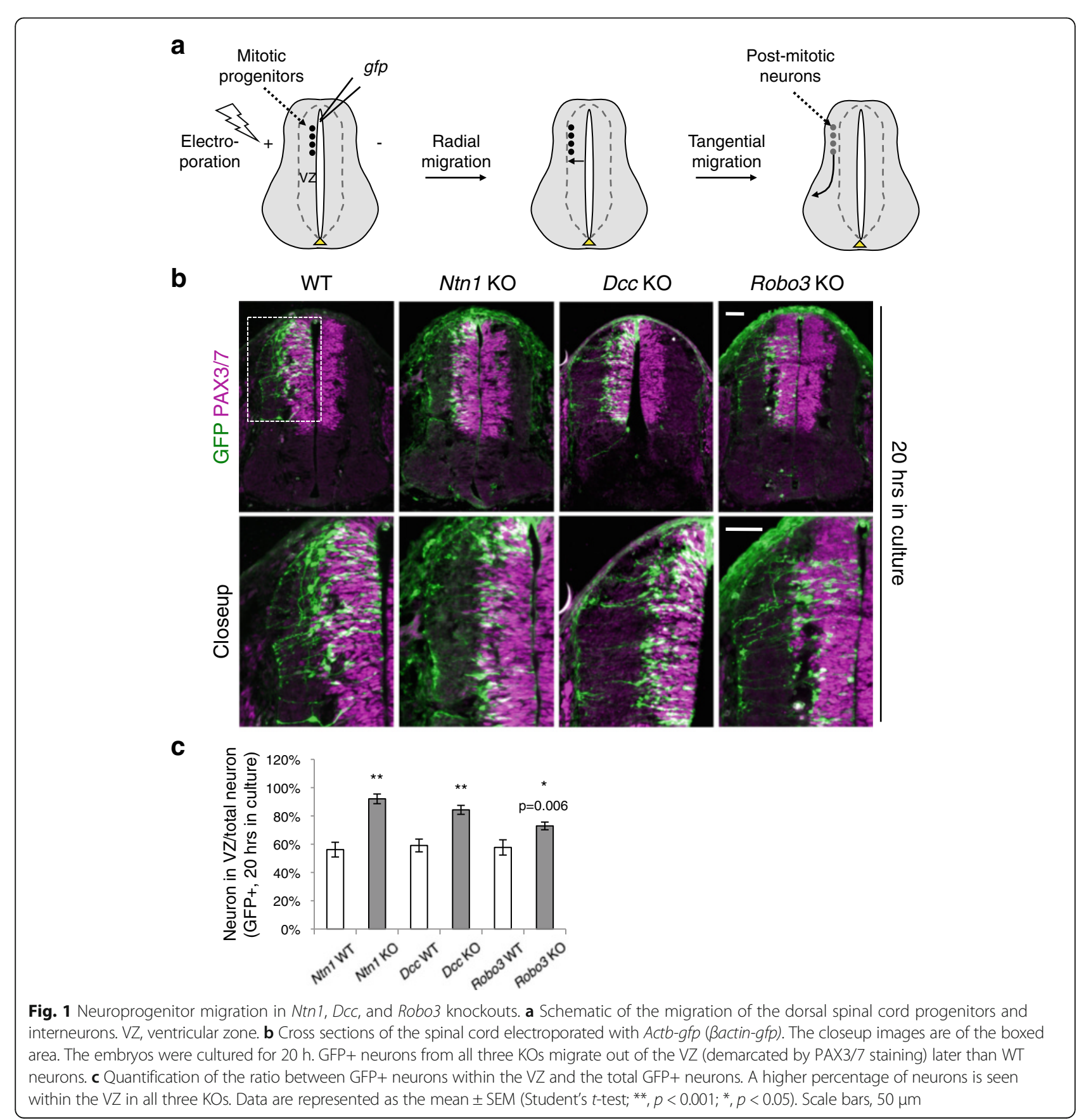


[18]. Thus, ROBO3 is critically important for commissural axon guidance. We wondered if $\mathrm{ROBO} 3$ is also involved in the earlier migration process.

In this study, we examined neuronal migration in Ntn1 and Robo3 KOs, in direct comparison with Dcc mutants. Using pulse labeling of the dorsal spinal cord progenitors, we found that the radial migration of these cells is delayed in Ntn1 and Robo3 KOs, as found previously in Dcc KOs. In addition, using immunohistochemistry of interneuron markers, we found that the tangential migration of dorsal interneurons is also delayed in all three mutants. Our data suggest that the Netrin1 ligand functions through DCC and $\mathrm{ROBO} 3$ receptors to promote the migration of the dorsal spinal cord neurons.

\section{Methods}

Mice

Ntn1, Dcc, Robo3, and Unc5c KOs were generated and described previously [19-22].

\section{Whole embryo culture}

The culture was carried out as previously described [23]. Embryos were electroporated at E9.5 with $g f p$ into one side of the spinal cord and were cultured for specified periods. The embryos were then fixed in $4 \%$ paraformaldehyde, cryopreserved in $30 \%$ sucrose, and embedded in OCT (optimal cutting temperature). $20 \mu \mathrm{m}$ transverse sections were collected and examined using fluorescent microscopy.

\section{Immunohistochemistry}

Immunohistochemistry was carried out as previously descried [24]. Antibodies used in the study include antiPAX3/7 (PA1-107, Thermo Fisher, raised against PAX3 and cross reacts with PAX7), anti-BARHL2 (NBP2-32013, Novus Biologicals), anti-LHX5 (AF6290, R\&D), anti-ISL1/2 (39.4D5, DSHB), anti-PAX6 (DSHB), anti-phosphoHistone H3 (9701, CST), anti-Ki67 (12202, CST), and anti-SOX2 (3728, CST).

\section{Quantification of phenotypes}

For phenotypic quantification in all experiments, KOs were compared with WT littermate controls. To minimize developmental variation, we used embryos of comparable sizes and examined spinal cord tissues from the brachial level. At least three embryos for each genotype and at least five sections from each embryo were quantified. In all phenotypic analyses, the defects were consistently seen in

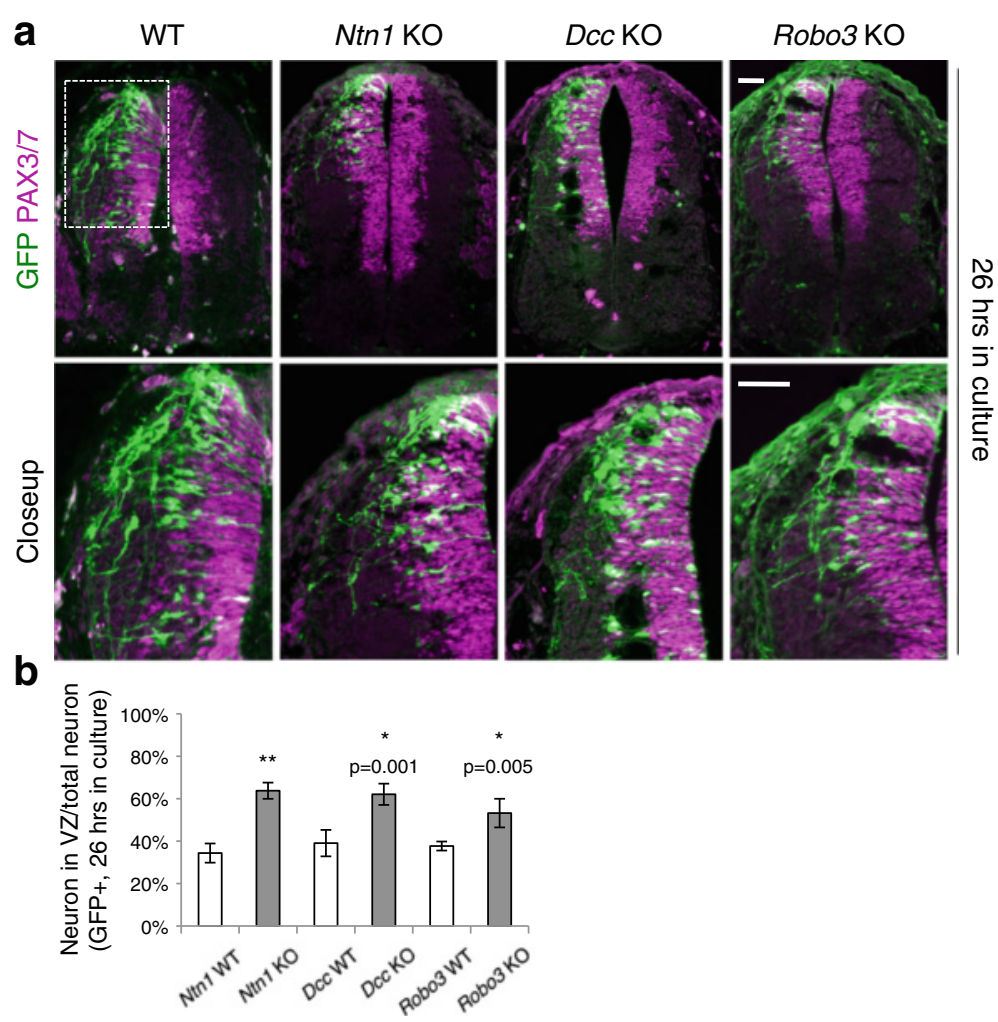

Fig. 2 Neuroprogenitor migration in Ntn1, Dcc, and Robo3 knockouts. a Cross sections of the spinal cord electroporated with Actb-gfp (Bactin-gfp). The closeup images are of the boxed area. The embryos were cultured for $26 \mathrm{~h}$. Some GFP+ neurons from the KOs are able to exit the VZ (PAX3/7+ area) after 26 h. $\mathbf{b}$ Quantification of the ratio between GFP+ neurons within the VZ and the total GFP+ neurons. A higher percentage of GFP+ neurons is seen within the VZ in the KOs than in controls. Data are represented as the mean \pm SEM (Student's t-test; **, $p<0.001 ;{ }^{*}, p<0.05$ ). Scale bars, $50 \mu \mathrm{m}$ 
all embryos examined. The severity of defects was comparable between animals and between different sections of the same embryo. Representative images are shown in all figures.

For quantifying the ventral migration of interneurons, the distance from the dorsal margin of the spinal cord to the ventral most BARHL2+ neurons (h1) is compared to the total height of the spinal cord (h2) (Figs. 6, 7). For the lateral migration, the distance between the medial most and lateral most BARHL2+ neurons (w1) is compared to the distance between the medial most neurons and the lateral margin of the spinal cord (w2). The distances were measured using Image (NIH, Bethesda, MD).

\section{Results}

By microinjection and electroporation, we introduced Actb-gfp (aka ßactin-gfp) into the progenitors adjacent to the lumen of the neural tube at E9.5 (Fig. 1a). During the subsequent culturing of the whole mouse embryos, the mitotic progenitors first migrated laterally away from the ventricle and can continue to divide [1-3]. At the onset of neurogenesis around E10 in mice, the progenitors began to exit the cell cycle and to migrate out of the VZ. After culturing, we labeled the VZ with antibodies against PAX3/7, which are expressed by the dorsal progenitors [1,
2], and examined the lateral movement of the GFP+ progenitors. In this assay, we previously observed that the dorsal progenitors are more frequently targeted than the ventral ones $[4,23]$, likely due to the fact that the dorsal progenitors are more actively proliferating and differentiating during the culture period. We first cultured the embryos for $20 \mathrm{~h}$ and examined the positions of GFP+ neurons. In WT embryos, many progenitors have exited the $\mathrm{VZ}$ and reached the lateral spinal cord. However, in Dcc KOs, the majority of GFP+ neurons were still positioned within the VZ ([4], Fig. 1b,c). Similarly, GFP+ progenitors in Ntn1 and Robo3 KOs were also mostly found within the VZ (Fig. 1b,c). We extended the culture period to $26 \mathrm{~h}$ to determine if the mutant progenitors are able to exit the VZ later. After the longer culture period, most WT neurons have exited the VZ and have extended axons toward the ventral half (Fig. 2). In comparison, neuroprogenitors in all three mutants were able to arrive at the lateral spinal cord, but the percentage of neurons within the VZ was still higher than in WT (Fig. 2). These results suggest that the radial migration of neuroprogenitors is likely to be delayed, but not completely blocked, in Ntn1, Dcc, and Robo3 KOs.

As the exit of neuroprogenitors from the VZ and their leaving the cell cycle are highly coordinated, the delay in

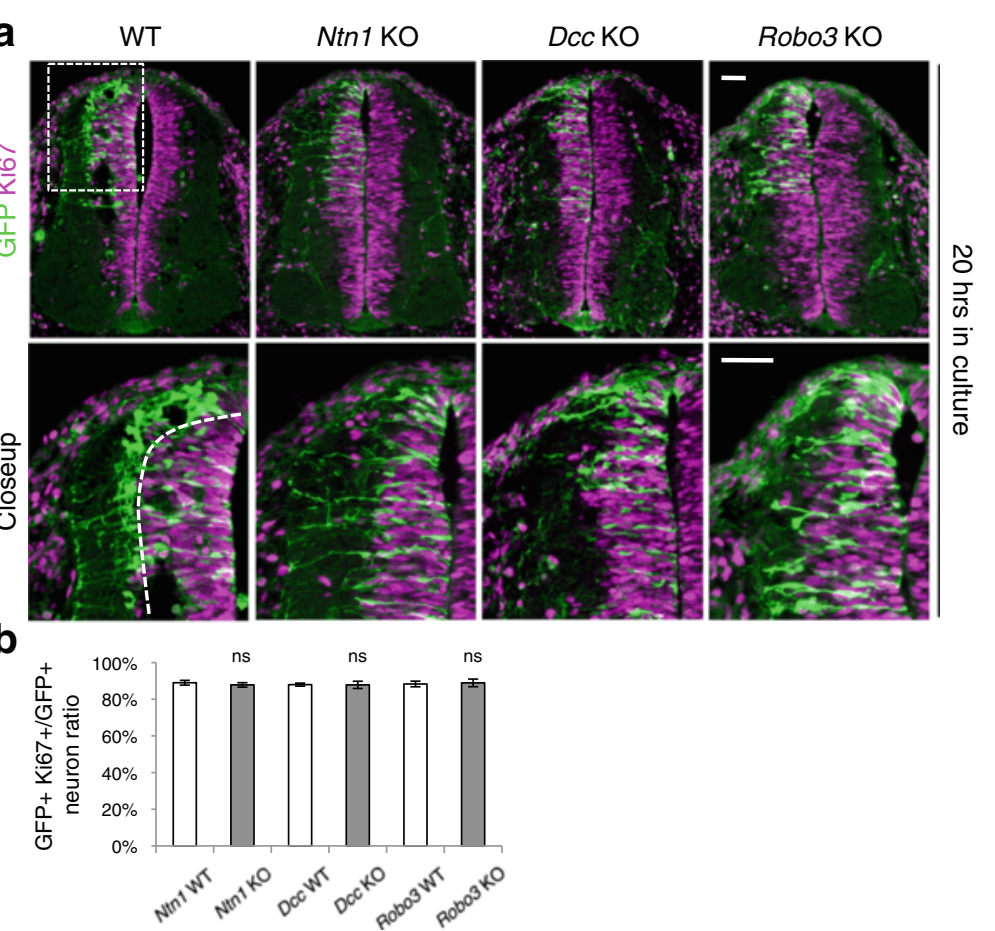

Fig. 3 Cell proliferation state in neuroprogenitors in Ntn1, Dcc, and Robo3 knockouts. a Cross sections of the spinal cord from embryos cultured for $20 \mathrm{~h}$. The closeup images are of the boxed area and the dashed line outlines the VZ. Anti-Ki67 staining marks neurons that are inside the cell cycle. $\mathbf{b}$ Quantification of the ratio between GFP+ Ki67+ neurons and the total GFP+ neurons within the VZ. The percentage of neurons that are inside the cell cycle is comparable between all three KOs and WT controls. Data are represented as the mean \pm SEM (Student's t-test; ns, not significant). Scale bars, $50 \mu \mathrm{m}$ 
reaching the lateral spinal cord can also result from a cell cycle defect. To examine this possibility, we studied the GFP+ neurons within the VZ using the Ki67 cell proliferation marker. We found that the percentage of neurons that are proliferating (i.e. Ki67+) is comparable between the three mutants and their respective controls (Fig. 3). These results suggest that the increase in GFP+ neuroprogenitors within the $\mathrm{VZ}$ is unlikely to result from a cell cycle abnormality.

Previously, we found that in $D c c$ KOs, the overall development of neuroprogenitors, except for their migration, is unaffected [4]. Using the same approaches, we further examined Ntn1 and Robo3 KOs. We analyzed cell proliferation using cell cycle markers, including

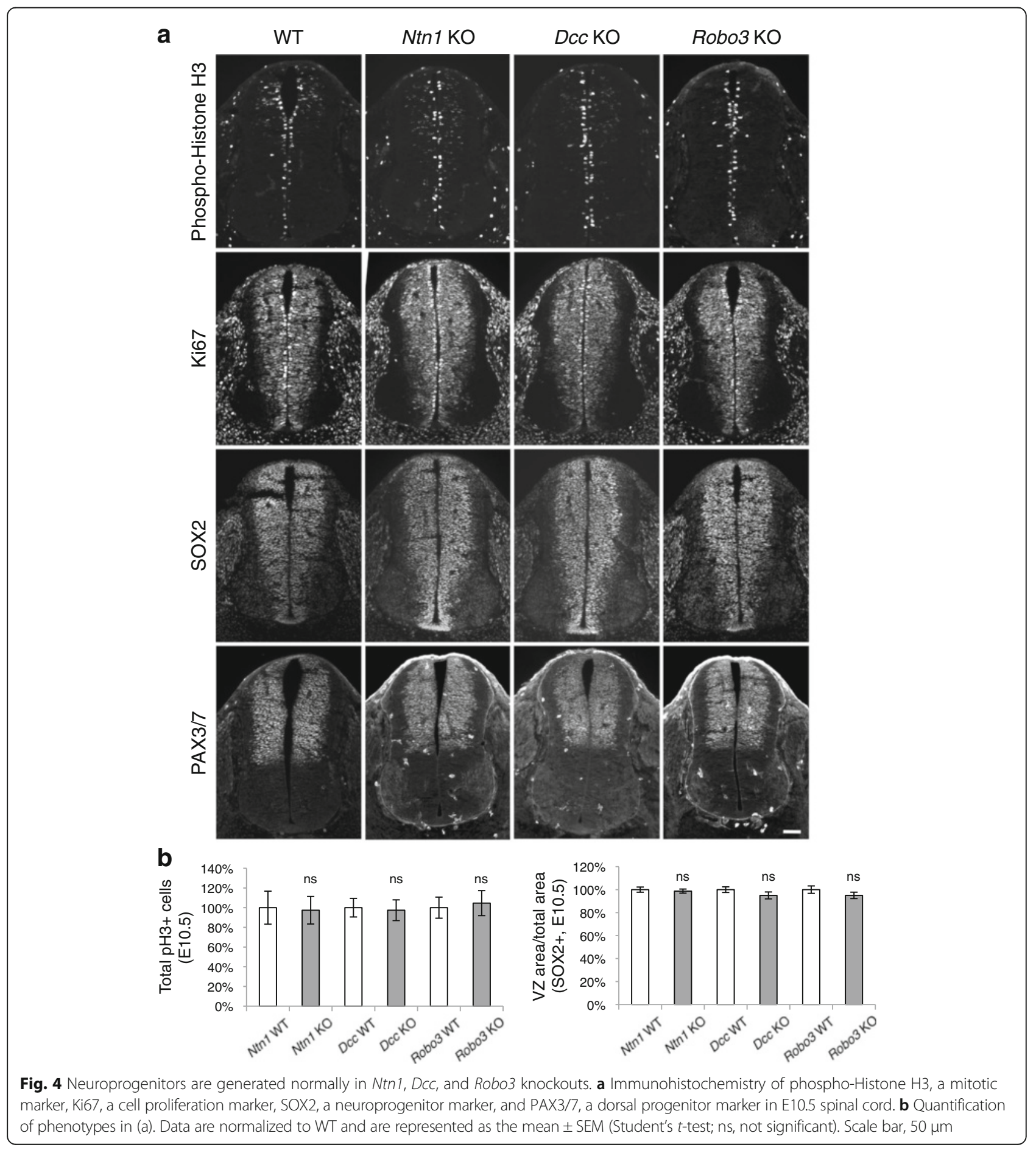


phospho-Histone H3 (pH3), a mitosis-specific marker, and Ki67. We found that the number of neural stem cells and progenitors is normal in Ntn1 and Robo3 mutants (Fig. 4). In addition, we labeled neuroprogenitors in the whole spinal cord with anti-SOX2 and the dorsal progenitors with anti-PAX3/7, and found no change in the localization or organization of these progenitor populations (Fig. 4). Thus, stem cells and progenitors are generated normally in the mutants.

We also examined if the differentiation of progenitors into interneurons and motor neurons is normal. Using the BARHL2, ISL1/2, and LHX5 markers, which are transcription factors expressed by different populations of neurons [1-3], we found that a normal number of neurons are born in Dcc KOs at E10.5 [4]. Using the same assays, we examined Ntn1 and Robo3 KOs and found that neither $\mathrm{KO}$ displayed any abnormalities in neuronal differentiation (Fig. 5). Ntn1 KOs were also reported to have a normal number of spinal cord neurons at E11.5 and E13.5 [21, 25]. Therefore, the loss of Ntn1, Dcc, or Robo3 does not affect neurogenesis, either.

Differentiated spinal cord neurons continue to migrate along mediolateral and dorsoventral axes to reach the final positions [1-3]. The dI1 population of interneurons are born at the dorsal margin of the spinal cord. They migrate both laterally and ventrally and give rise to contralateral- and ipsilateral-projecting subpopulations (dI1c and dI1i, respectively). Using anti-BARHL2 to follow the tangential migration of dI1 neurons [26], we previously found that both mediolateral and dorsoventral migration is delayed in Dcc KOs [4]. We further examined Ntn1 and Robo3 KOs. As discussed above, a normal number of BARHL2+ neurons are born at E10.5 in the mutants (Fig. 5). By E11.75, most neurons in WT have migrated ventrally and some start to arrive at the lateral margin of the spinal cord ([26], Fig. 6a). In
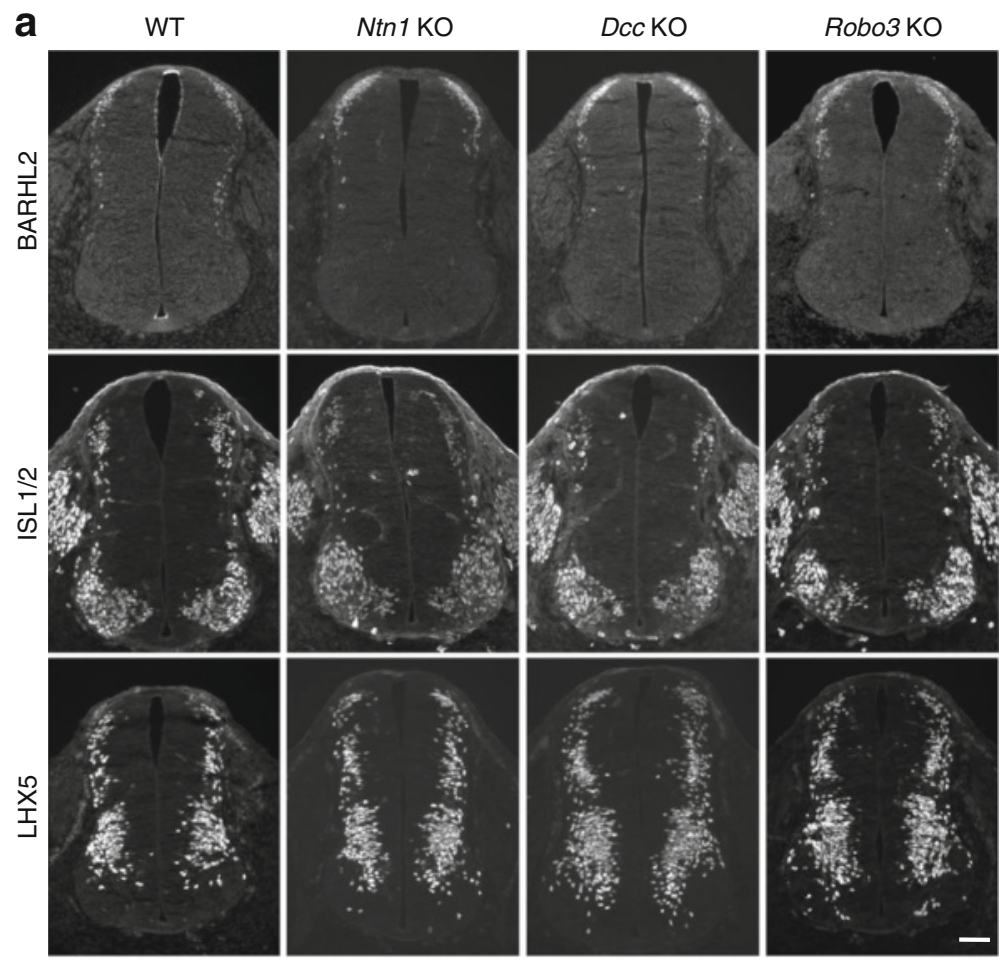

b
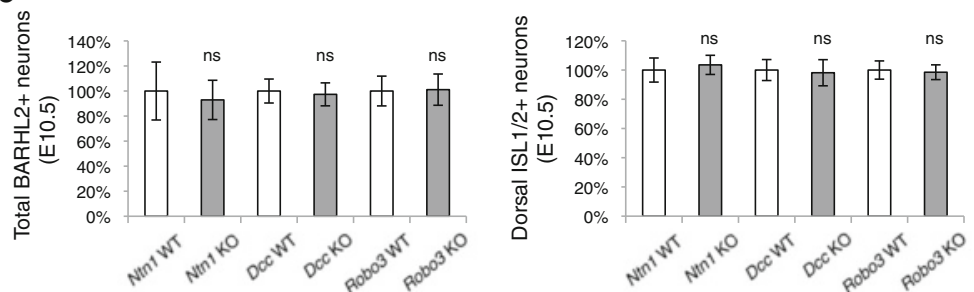

Fig. 5 Neuronal differentiation is normal in Ntn1, Dcc, and Robo3 knockouts. a Immunohistochemistry of BARHL2, ISL1/2, and LHX5, in E10.5 spinal cord. The markers are expressed by different populations of interneurons and motor neurons. b Quantification of BARHL2+ neurons and ISL1/2+ dorsal interneurons. Data are normalized to WT and are represented as the mean \pm SEM (Student's $t$-test; ns, not significant). Scale bar, $50 \mu m$ 


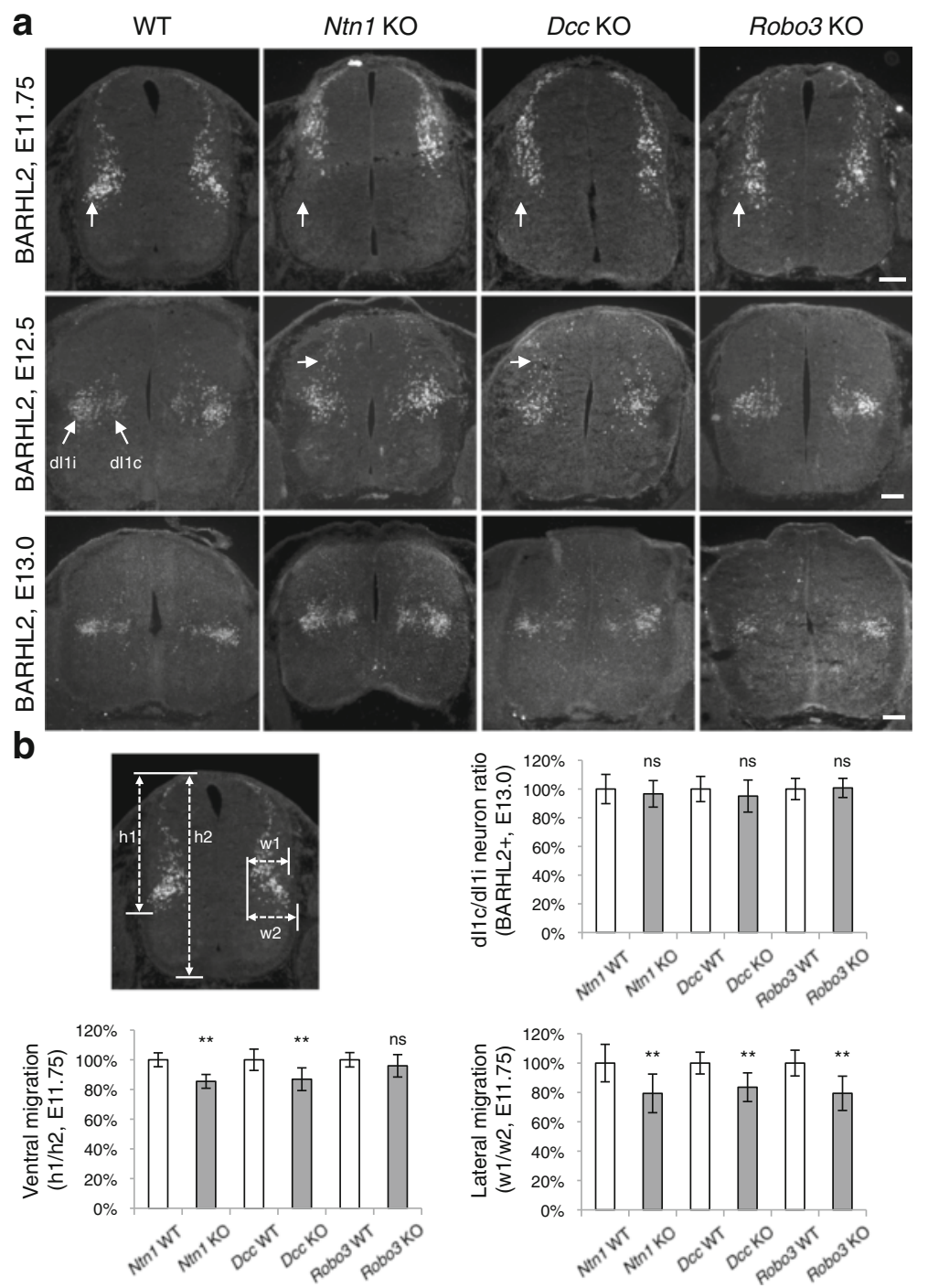

Fig. 6 Dorsal interneuron migration is delayed in Ntn1, Dcc, and Robo3 knockouts. a Immunohistochemistry of BARHL2 at different stages. At E11.75, BARHL2+ neurons fail to reach the lateral spinal cord in the KOs (indicated by arrow). Their ventral migration is also delayed. At E12.5, dl1i (ipsilateral) and dl1c (contralateral) populations appear normal in WT and Robo3 KOs. There are still some neurons in the dorsal spinal cord in Ntn1 and Dcc KOs (arrow). At E13.0, all mutants have two distinct populations of dl1 neurons. b Quantification of phenotypes in (a). For quantifying the ventral migration, the distance from the dorsal margin to the ventral most BARHL2+ neurons ( $h 1$ ) is compared to the total height of the spinal cord (h2). For the lateral migration, the distance between the medial most and lateral most BARHL2+ neurons (w1) is compared to the distance between the medial most neurons and the lateral margin of the spinal cord (w2). The distances were measured using ImageJ. Data are normalized to WT and are represented as the mean \pm SEM (Student's $t$-test; ${ }^{*}, p<0.001$; ns, not significant). Scale bars, $50 \mu \mathrm{m}$

contrast, in Ntn1, Dcc, and Robo3 KOs, BARHL2+ neurons were located more medially and dorsally than normal (Fig. 6). Later at E12.5, the dI1c and dI1i populations can be well discerned in WT, with dI1i residing at a more lateral position ([26], Fig. 6a). Similarly, Robo3 KOs had two distinct subpopulations (Fig. 6). Ntn1 and Dcc KO neurons appeared to be slightly more medially and dorsally positioned. At E13.0, dI1c and dI1i could be seen in all three mutants and the ratio between them was normal (Fig. 6). Therefore, there is a delay in the tangential migration of dorsal interneurons in all three mutants, but both contralateral and ipsilateral neurons are eventually differentiated. The delay is less severe in Robo3 KOs than in Dcc KOs, consistent with previous observations in commissural axonal outgrowth [18]. Ntn1 KO, on the other hand, caused a more severe defect than $D c c \mathrm{KO}$, also consistent with previous observations in axon guidance [21,25]. This is likely due to the fact that another homologue of DCC, the Neogenin (Neo1) receptor, has some redundant function and thus could compensate for the loss of DCC [24]. 
The UNC5 family of receptors also bind Netrin and can act as DCC coreceptors. UNC5s by themselves or in complex with DCC mediate axonal repulsion [9]. UNC5s have not been shown to function in spinal commissural axons. In addition, Ntn1 null mutants display rather distinct phenotypes from the knockout of the Unc5 family receptors, suggesting that Netrin1 is not the main ligand for UNC5s in vivo [21]. For comparison, we also examined Unc5c KOs for the migration phenotype using the BARHL2 marker. At E11.75, when Ntn1, Dcc, and Robo3 KOs displayed a migration delay, Unc5c KOs did not have such a defect and the BARHL2+ neurons have reached comparable positions as in WT controls (Fig. 7). Taken together, the UNC5 repulsive receptors are unlikely to be involved in the same migration process as Netrin1 and DCC.

By in situ hybridization and immunohistochemistry, $D c c$ expression is observed in interneurons and motor neurons, as well as in neuroprogenitors $[4,5,27,28]$. Robo3 expression is more specific and mostly within commissural interneurons [20, 23]. Using specific antibodies, we found that $\mathrm{ROBO} 3$ protein is also present on the periphery of dorsal progenitors (Additional file 1: Figure S1). Therefore, $D c c$ and Robo3 are most likely to function cell-autonomously within progenitors and interneurons during migration, as they do during axon guidance.

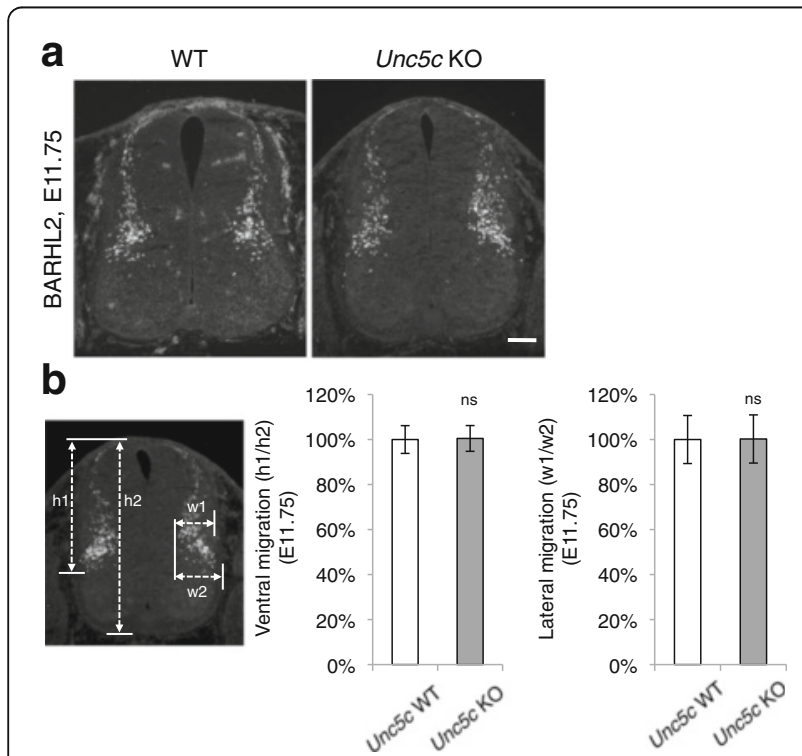

Fig. 7 dl 1 interneuron migration is normal in Unc5c knockouts. a BARHL2+ immunohistochemistry in WT and Unc5c KO spinal cord at E11.75. The dorsal interneurons have arrived at the normal positions. b Quantification of neuronal migration (see Fig. 6 for description of quantification). Data are normalized to WT and are represented as the mean \pm SEM (Student's $t$-test; ns, not significant). scale bar, $50 \mu \mathrm{m}$

\section{Discussion}

Neuronal migration is one of the early and critical steps of neural development. It is a complex cellular process involving many classes of molecules, including extracelluar ligands and transmembrane receptors, intracellular signaling molecules, cytoskeletal and motor proteins, and transcriptional factors [29-32]. Within the developing spinal cord, the molecular mechanism underlying the migration of neuroprogenitors and differentiated neurons is mostly uncharacterized. The Reelin/VLDLR/ApoER2 pathway has been shown to regulate the migration of certain populations in the ventral spinal cord [33]. Our previous study reveals that DCC is important for the migration of the dorsal neuroprogenitors and interneurons [4]. DCC has also been shown to promote the ventral migration of several classes of dorsal spinal cord interneurons [5]. Built upon these findings, we extended the study to the Netrin1 ligand and the ROBO3 coreceptor, which act together with DCC to mediate axonal attraction. Interestingly, the loss of any of these three molecules reduces the radial migration of neuroprogenitors and the tangential migration of dorsal interneurons. In contrast, loss of the UNC5C receptor does

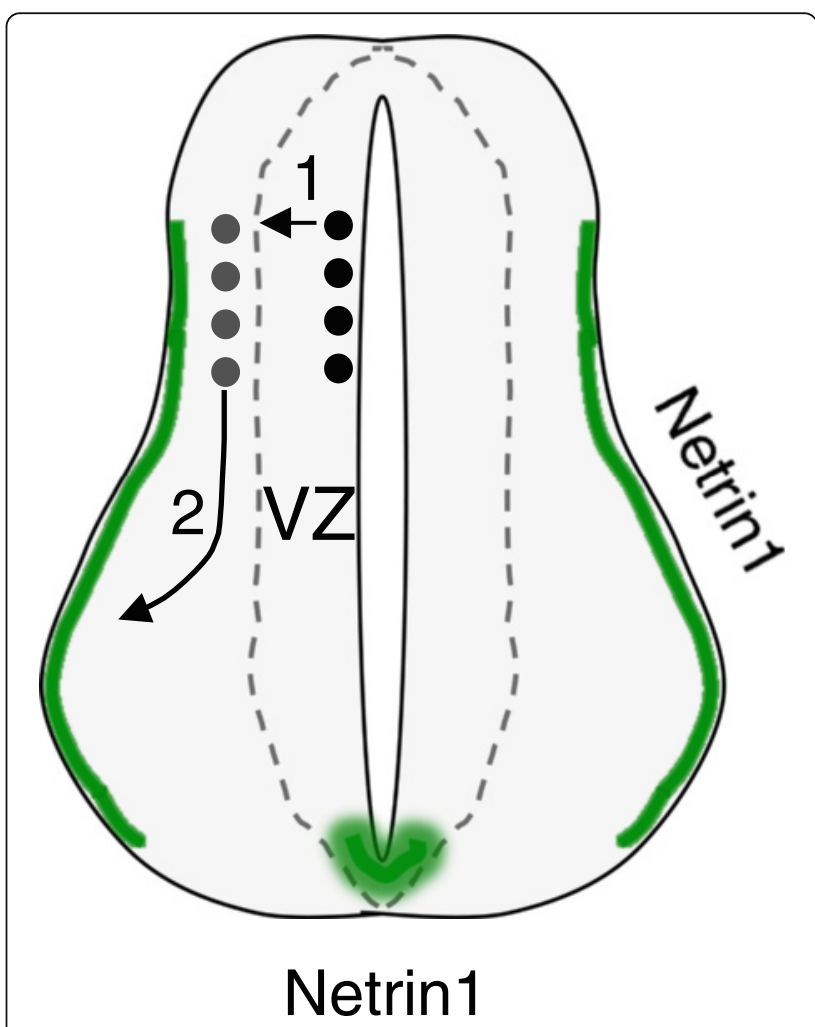

Fig. 8 Model of Netrin1/DCC signaling during neuronal migration in the dorsal spinal cord. Netrin 1 protein is enriched at the ventral midline and at the lateral margin of the spinal cord (green areas). It acts through DCC and $\mathrm{ROBO} 3$ receptors to attract the neurons both laterally and ventrally. 1, radial migration of neuroprogenitors; 2 , tangential migration of dorsal interneurons; VZ, ventricular zone 
not cause such a delay. Given the enrichment of Netrin1 at the lateral and ventral margin of the spinal cord [13, 14], our results support a model that Netrin1/DCC signaling also attracts the migration of the dorsal spinal cord progenitors and neurons (Fig. 8).

Our experimental approach does not allow us to efficiently target the ventral spinal cord progenitors in cultured mouse embryos. We thus cannot determine if Netrin1/ DCC also acts in the ventral populations. Within the ventrally located motor neurons, Netrin1/DCC as well as the SLIT/ROBO pathway have been shown to be important for the dorsoventral positioning of the cell bodies relative to the midline [34]. The loss of the Netrin1/DCC signaling leads to motor neurons positioning in a more dorsal position than normal [34]. Therefore, Netrin1/DCC is likely to be involved in the migration of additional spinal cord neurons. Robo3 is not expressed in the ventral spinal cord, except in the ventral most V3 interneurons [20, 23], and is thus unlikely to play a role in most ventral neurons.

The fact that in Ntn1, Dcc, and Robo3 KOs, neuronal migration is delayed but not completely blocked suggests that there may be additional molecules at play. In addition, although the early-born dorsal interneurons (generated from E10 to around E11.5) migrate predominantly ventrally, late-born populations (generated around E12) migrate dorsally $[1,2]$. It is thus likely that a distinct pathway directs the dorsal migration. Additional studies are warranted to unveil the identity of these molecules.

\section{Additional file}

Additional file 1: Figure S1. $\mathrm{ROBO} 3$ expression in the spinal cord. $\mathrm{ROBO} 3$ is highly expressed in differentiated commissural neurons and their axons, and can also be detected at the periphery of progenitors. PAX7 labels the dorsal progenitors. PAX6 labels progenitors except in the ventral most spinal cord. Scale bars, $50 \mu \mathrm{m}$ in the top panel and $10 \mu \mathrm{m}$ in the bottom. (PDF $7076 \mathrm{~kb})$

\section{Abbreviations}

ApoER2: Apolipoprotein E receptor 2; DCC: Deleted in colorectal carcinoma; KO: Knockout; Ntn1: Netrin1; Robo: Roundabout; Unc: Uncoordinated; VLDLR: Very low density lipoprotein receptor; VZ: Ventricular zone; WT: Wild type

\section{Acknowledgements}

We thank Daniel Morales and Dr. Artur Kania for providing the Unc5c KO embryos, and Caleb Anderson for technical support.

\section{Funding}

This work was supported by the National Institutes of Health ( $\mathrm{NIH})$ R01EY024261 (to HJ), R21DC014916 (to LVG), and F31DC014603 (to ARY) and by the Boettcher Foundation (to ZC).

\section{Availability of data and material}

The original data and quantification analyses in this study are available from the corresponding authors upon request.

\section{Authors' contributions}

H.J.J. and Z.C. designed the study. Z.C. performed the experiments. A.R.Y. and L.V.G. provided reagents. Z.C. analyzed the data. H.J.J. and Z.C. wrote the paper with contributions from the other authors. All authors read and approved the final manuscript.

\section{Competing interests}

The authors declare that they have no competing interests.

\section{Consent for publication}

Not applicable.

\section{Ethics approval}

All experimental manipulations and care of mice have been approved by the University of Colorado Boulder Institutional Animal Care and Use Committee (protocol number 1310.02).

\section{Author details}

'Department of MCDB, University of Colorado, Boulder, CO 80309, USA ${ }^{2}$ Department of Neurobiology, Harvard Medical School, Boston, MA 02115, USA.

Received: 11 August 2016 Accepted: 17 October 2016

Published online: 26 October 2016

\section{References}

1. Caspary T, Anderson KV. Patterning cell types in the dorsal spinal cord: what the mouse mutants say. Nat Rev Neurosci. 2003:4:289-97.

2. Helms AW, Johnson JE. Specification of dorsal spinal cord interneurons. Curr Opin Neurobiol. 2003;13:42-9.

3. Lu DC, Niu T, Alaynick WA. Molecular and cellular development of spinal cord locomotor circuitry. Front Mol Neurosci. 2015;8:25.

4. Leggere JC, Saito Y, Darnell RB, Tessier-Lavigne M, Junge HJ, Chen Z. NOVA regulates Dcc alternative splicing during neuronal migration and axon guidance in the spinal cord. Zoghbi HY, editor. eLife. 2016;5:e14264.

5. Ding Y-Q, Kim J-Y, Xu Y-S, Rao Y, Chen Z-F. Ventral migration of early-born neurons requires Dcc and is essential for the projections of primary afferents in the spinal cord. Development. 2005;132:2047-56.

6. Culotti JG, Merz DC. DCC and netrins. Curr Opin Cell Biol. 1998:10:609-13.

7. Cooper HM, Gad JM, Keeling SL. The Deleted in Colorectal Cancer netrin guidance system: a molecular strategy for neuronal navigation. Clin Exp Pharmacol Physiol. 1999;26:749-51.

8. Killeen MT. The dual role of the ligand UNC-6/Netrin in both axon guidance and synaptogenesis in C. elegans. Cell Adh Migr. 2009;3:268-71.

9. Moore SW, Tessier-Lavigne M, Kennedy TE. Netrins and their receptors. Adv Exp Med Biol. 2007:621:17-31.

10. Dickson BJ, Zou Y. Navigating intermediate targets: the nervous system midline. Cold Spring Harb Perspect Biol. 2010;2:a002055.

11. Dickson BJ. Molecular mechanisms of axon guidance. Science. 2002;298:1959-64.

12. Evans TA, Bashaw GJ. Axon guidance at the midline: of mice and flies. Curr Opin Neurobiol. 2010;20:79-85.

13. MacLennan AJ, McLaurin DL, Marks L, Vinson EN, Pfeifer M, Szulc SV, et al. Immunohistochemical Localization of Netrin-1 in the Embryonic Chick Nervous System. J Neurosci. 1997;17:5466-79.

14. Kennedy TE, Wang H, Marshall W, Tessier-Lavigne M. Axon Guidance by Diffusible Chemoattractants: A Gradient of Netrin Protein in the Developing Spinal Cord. J Neurosci. 2006;26:8866-74.

15. Laumonnerie C, Da Silva RV, Kania A, Wilson SI. Netrin 1 and Dcc signalling are required for confinement of central axons within the central nervous system. Development. 2014;141:594-603.

16. Watanabe K, Tamamaki N, Furuta T, Ackerman SL, Ikenaka K, Ono K. Dorsally derived netrin 1 provides an inhibitory cue and elaborates the 'waiting period' for primary sensory axons in the developing spinal cord. Development. 2006;133:1379-87

17. Guthrie S. Axon guidance: mice and men need Rig and Robo. Curr Biol. 2004; 14:R632-4.

18. Zelina P, Blockus H, Zagar Y, Peres A, Friocourt F, Wu Z, et al. Signaling switch of the axon guidance receptor Robo3 during vertebrate evolution. Neuron. 2014:84:1258-72.

19. Fazeli A, Dickinson SL, Hermiston ML, Tighe RV, Steen RG, Small CG, et al. Phenotype of mice lacking functional Deleted in colorectal cancer (Dcc) gene. Nature. 1997;386:796-804. 
20. Sabatier C, Plump AS, Le M, Brose K, Tamada A, Murakami F, et al. The divergent Robo family protein rig-1/Robo3 is a negative regulator of slit responsiveness required for midline crossing by commissural axons. Cell. 2004;117:157-69.

21. Yung AR, Nishitani AM, Goodrich LV. Phenotypic analysis of mice completely lacking netrin 1. Dev Camb Engl. 2015;142:3686-91.

22. Ackerman SL, Kozak LP, Przyborski SA, Rund LA, Boyer BB, Knowles BB. The mouse rostral cerebellar malformation gene encodes an UNC-5-like protein. Nature. 1997;386:838-42

23. Chen Z, Gore BB, Long H, Ma L, Tessier-Lavigne M. Alternative splicing of the Robo3 axon guidance receptor governs the midline switch from attraction to repulsion. Neuron. 2008;58:325-32.

24. Xu K, Wu Z, Renier N, Antipenko A, Tzvetkova-Robev D, Xu Y, et al. Structures of netrin-1 bound to two receptors provide insight into its axon guidance mechanism. Science. 2014:344:1275-9.

25. Bin JM, Han D, Lai Wing Sun K, Croteau L-P, Dumontier E, Cloutier J-F, et al. Complete Loss of Netrin-1 Results in Embryonic Lethality and Severe Axon Guidance Defects without Increased Neural Cell Death. Cell Rep. 2015;12:1099-106.

26. Ding Q, Joshi PS, Xie Z, Xiang M, Gan L. BARHL2 transcription factor regulates the ipsilateral/contralateral subtype divergence in postmitotic dl1 neurons of the developing spinal cord. Proc Natl Acad Sci U S A. 2012;109:1566-71.

27. Keino-Masu K, Masu M, Hinck L, Leonardo ED, Chan SS, Culotti JG, et al. Deleted in Colorectal Cancer (DCC) encodes a netrin receptor. Cell. 1996;87:175-85

28. Phan KD, Croteau LP, Kam JW, Kania A, Cloutier JF, Butler SJ. Neogenin may functionally substitute for Dcc in chicken. PLoS One. 2011;6:e22072.

29. Dantas TJ, Carabalona A, Hu DJK, Vallee RB. Emerging roles for motor proteins in progenitor cell behavior and neuronal migration during brain development. Cytoskeleton. 2016;73:566-76.

30. Ohtaka-Maruyama C, Okado H. Molecular Pathways Underlying Projection Neuron Production and Migration during Cerebral Cortical Development. Front Neurosci. 2015;9:447.

31. Kawauchi T. Cellullar insights into cerebral cortical development: focusing on the locomotion mode of neuronal migration. Front Cell Neurosci. 2015;9:394.

32. Hippenmeyer S. Molecular Pathways Controlling the Sequential Steps of Cortical Projection Neuron Migration. In: Nguyen L, Hippenmeyer S, editors. Cell. Mol. Control Neuronal Migr. [Internet]. Dordrecht: Springer Netherlands; 2014. p. 1-24. Available from: http://dx.doi.org/10.1007/978-94-007-7687-6_1.

33. Vaswani AR, Blaess $S$. Reelin Signaling in the Migration of Ventral Brain Stem and Spinal Cord Neurons. Front Cell Neurosci. 2016;10:62

34. Kim M, Fontelonga T, Roesener AP, Lee H, Gurung S, Mendonca PR, et al. Motor neuron cell bodies are actively positioned by Slit/Robo repulsion and Netrin/DCC attraction. Dev Biol. 2015;399:68-79.

\section{Submit your next manuscript to BioMed Central and we will help you at every step:}

- We accept pre-submission inquiries

- Our selector tool helps you to find the most relevant journal

- We provide round the clock customer support

- Convenient online submission

- Thorough peer review

- Inclusion in PubMed and all major indexing services

- Maximum visibility for your research

Submit your manuscript at www.biomedcentral.com/submit 